MARTINA WERNER

Substantivierter Infinitiv statt Derivation.

Ein ,echter' Genuswechsel und ein Wechsel der Kodierungstechnik

innerhalb der deutschen Verbalabstraktbildung

\section{HELLMUT SPIEKERMANN}

Pronominaladverbien im Niederdeutschen und in

der norddeutschen Regionalsprache

VILMOS ÁGEL

+/-Wandel. Am Beispiel der Relativpartikeln so und wo

\section{DAGMAR BITTNER}

Die deutsche Klammerstruktur:

Epiphänomen der syntaktischen Realisierung von Assertion und

Thema-Rhema-Gliederung

\section{WERNER ABRAHAM}

Methodische Überlegungen zu Grammatikalisierung,

zyklischem Wandel und dem Wechsel von Analytik zu Synthetik und zyklisch weiter zu Analytik (?)

Sachregister

Personenregister

\section{Damaris Nübling}

\section{Lässt sich ein Syntheseindex erstellen? Zur Problematisierung und Präzisierung eines (allzu) geläufigen Begriffs}

\section{Abstract}

In descriptions of German language history, it is almost a commonplace to characterize German as a language developing towards analytic structures (due to, e.g., the rise of articles, of obligatory subject pronouns, or of periphrastic constructions). Obviously opposite developments such as the cliticization of preposition and article, the fragmentation of the ablaut system (leading to an increase of allomorphy) or the diffusion of the motphological umlaut usually ate not considered in this debate. In addition, it is never precisely defined what analytic structures exactly consist of. In this article, which is based on the approach of Schwegler (1990), the phenomenon of analyticity and syntheticity is specified semantically, syntactically, morphologically and phonologically and, at the same time, from a syntagmatic and a paradigmatic perspective. The so-called combinatory expression type which is typical for German inflection is identified as a strategy leading to a high degree of syntheticity. This article concludes that there is not much evidence that German has become a mote analytic language.

\section{Das Problem}

Es handelt sich fast um einen sprachhistorischen Topos, wenn davon die Rede ist, dass sich das Deutsche von einer synthetischen zu einer analytischen Sprache entwickelt habe, oder zumindest zu einer analytischeren oft hat man das Englische im Blick, das den isolierenden Sprachen nahèstehen soll. Die Darstellungen zur deutschen Sprachgeschichte sind voll von diesem Topos, und anscheinend ist dieses Konzept intuitiv so eingängig, dass kaum hinterfragt wird, was man denn genau unter synthetischen bzw. analytischen Strukturen zu verstehen habe. Als ein Beispiel unter vielen sei aus der Einführung „Deutsche Sprache gestern und heute" von Astrid Stedje (1999) zitiert:

In den germanischen Sprachen lässt sich von ältester Zeit bis heute die Entwicklung von einem stark synthetischen zu einem analytischeren Sprachbau verfolgen: Das Ahd. verfügt noch über ein reiches Endungssystem und konnte durch dieses grammatische Beziehungen ausdrücken, für die wir heute beschreibende Funktionswörter benötigen [...] Nachdem aber das Endungssystem undeutlich geworden war, mussten Genus und Kasus auf andere Art angezeigt werden. Im heutgen Deutsch geschieht dies durch den bestimmten und unbestimmten Artikel, attributive Pronomina und stark flektierende Adjektive [...]. (Stedje 1999: 18-19) 
Einige Seiten später liest man:

Die Schwächung der unbetonten Silben im Germanischen trug zum Verfall des überreichen indogermanischen Formensystems bei. Hier beginnt die Entwicklung vom synthetischen zum analytischen Sprachbau. (Stedje 1999: 47)

Auch in tiefer gehenden Darstellungen findet sich dieser Gedanke, z.B. in Sonderegger (1979), Schmidt (1998), Polenz (2000), Schmidt (2007), Besch \& Wolf (2009) etc. Als gängige Synthese- > Analyse-Phänomene im Deutschen werden gemeinhin die folgenden betrachtet:

1. Ahd. Kasusendungen am Substantiv werden sukzessive abgebaut; stattdessen übernehmen nach und nach Artikel und Präpositionen den Ausdruck dieser vormaligen Kasusinformationen.

2. Derzeit erregt der angebliche Verlust des synthetischen Genitivs die Gemüter: Anstelle von meiner Mutter Hund oder der Hund meiner Mutter kommt es zu von-Umschreibungen (der Hund von meiner Mutter) bzw. zur sog. possessiven Dativumschreibung (meiner Mutter ibr Hund).

3. Der Verlust der Person/Numerus-Endungen am Verb hat angeblich zur Grammatikalisierung obligatorischer, analytischer Subjektspronomen geführt.

4. Der oberdeutsche Präteritumschwund hat zur Generalisierung von Perfekt, Plusquamperfekt und sog. Hyperperiphrasen geführt: sagte $>$ bat $(t e)$ gesagt $>$ bat $(t e)$ gesagt gebabt.

5. Auch der synthetische Konjunktiv schwindet und wird durch die würde-Umschreibung ersetzt: bülfe/bälfe $>$ würde belfen (zu äußerst interessanten Übergangsphänomenen in diesem Prozess s. Bittner \& Köpcke in diesem Band).

6. Schließlich wird auch die Entstehung des werden-Futurs im Frühnhd. sowie des sein- und werden-Passivs zur analytischen Drift des Deutschen gerechnet.

Im Folgenden geht es darum, den Begriff von Synthetizität bzw. Analytizität schärfer zu fassen, indem es diesen von mehreren Seiten her zu beleuchten gilt. Dabei wird in erster Linie auf Armin Schwegler (1990) "Analyticity and Syntheticity“ Bezug genommen, der diese Begrifflichkeit m.E. entscheidend geschärft und damit auch hinterfragt hat. Sein Gegenstand sind die romanischen Sprachen, vor allem das Französische. Die hier angestellten Überlegungen sollen, soweit möglich, auf das Deutsche übertragen werden. Es wird dabei auch deutlich, dass es im Deutschen Kodierungstechniken wie das kombinierende Verfahren gibt, die über die üblichen Verfahren in den romanischen Sprachen hinausgehen. Hinzu kommt eine Reihe weiterer Kriterien, die m.E. bei der Analyse/Synthese-Debatte zu berücksichtigen sind und Thema dieses Beitrags sind.
Thorsten Roelcke hat 2002 die (nicht neue) Idee geäußert, für das Deutsche einen sog. Syntheseindex zu erstellen. Sein Eindruck ist dabei, dass dieser ,im Deutschen nicht ab[nimmt] (wie von der Strömungstheorie postuliert), sondern [...] vergleichsweise unverändert [bleibt]“" (Roelcke 2002: 341). Es spricht tatsächlich vieles dafür, dass das Deutsche insgesamt weniger analytisch geworden ist, als ihm in sprachgeschichtlichen Darstellungen attestiert wird. Auch auf diese Frage, die allerdings die Messbarkeit von Synthetizität voraussetzt, wird im Folgenden einzugehen sein.

\section{Analytizität bzw. Synthetizität als syntagmatisches Phänomen}

Die meisten LinguistInnen verorten Analytizität bzw. Synthetizität auf det syntagmatischen Achse und verstehen in aller Regel darunter, dass grammatische Informationen entweder morphologisch "am Wort", d.h. an einem Lexem bzw., wenn man Pronomen oder Artikel betrachtet, an einem Grammem markiert werden, oder dass sie syntaktisch, d.h. über eigene (Funktions-)Wörter realisiert werden. In jedem Fall werden die Informationen mehr oder weniger fusionierend bzw., in den Worten von Ronneberger-Sibold (1980) und Werner (1987, 1989), komprimierend ausgedrückt. Klassisches Beispiel sind die im Nhd. obligatorischen Subjektpronomen,_die angeblich die ahd. Person/Numerussuffixe_am Verb ersetzt haben sollen. Dies stellt Abb. 1 dar.

\begin{tabular}{|c|c|}
\hline $\begin{array}{l}\text { synthetisch } \\
\text { + fusionierend } \\
\text { komprimierend }\end{array}$ & $\begin{array}{r}\text { analytisch } \\
\text {-fusionierend } \\
\text { expandierend }\end{array}$ \\
\hline $\begin{array}{ll}\text { ahd. } & \text { sing- } u \\
& \text { sing-is }(t) \\
& \text { sing-it }\end{array}$ & $\begin{aligned} \text { nhd. } & \text { ich sing-e } \\
& d u \text { sing-st } \\
& \text { sie/ er sing-t }\end{aligned}$ \\
\hline
\end{tabular}

Abbildung 1: Von analytisch zu synthetisch als syntagmatisches Phänomen

Abb. 1 zeigt die für das Deutsche üblicherweise angenommene Drift von links nach rechts und liefert ein Beispiel, das auf den ersten Blick einleuchtet: Was das Ahd. durch vollvokalische Verbflexive regressiv (postdeterminierend) ausdrückt, entwickelt sich zum Nhd. hin zu einem syntaktischpräponierten, progressiven Verfahren, den obligatorischen Subjektspronomen. Abgesehen davon, dass auch die heutigen Endungen noch größtenteils differenzieren, ist in Rechnung zu stellen, dass zwischen den prä- 
ponierten Pronomen und dem Verb Kongruenz besteht. Damit kommen wir zu der Frage, ob Kongruenz den Grad an Synthetizität steigert oder verringert. In Abschnitt 2.4 wird für eine Steigerung argumentiert.

\subsection{Zum Syntheseindex nach Greenberg (1954/1960)}

Einen einfachen syntagmatischen Synthesebegriff, der Schule gemacht hat, vertritt Greenberg (1954/1960) in „A quantitative approach to the morphological typology of language" "Hier versucht er, für typologische Zwecke den morphologischen Synthesegtad verschiedener Sprachen zu quantifizieren und damit vergleichbar zu machen. Die Formel ist denkbar einfach und genau dadurch breit anwendbar: Der Syntheseindex S ergibt sich aus der Zahl der Morpheme M, die durch die Zahl der Wörter W geteilt wird $(S=M / W)$. So besteht engl. $\{l o v\}-\{e d\}$ aus zwei Morphemen, aber aus nur einem Wort, was einen Syntheseindex von 2,0 ergibt. Isolierende (analytische) Sprachen müssen folglich einen Index von 1,0 haben. Ein synthetischer Sprachbau beginnt ab 2,0, ab einem Quotienten von 3,0 spricht Greenbetg von polysynthetischen Sprachen.

Es liegt nahe, dass diese Rechnung Probleme in sich birgt, angefangen bei der Definition, Zählung und Segmentierung der Morpheme sowie beim Wortbegtiff, für dessen Definition oft nicht einmal für ein und dieselbe Sprache Konsens besteht, geschweige denn interlingual. Auch stellt sich die Frage, wieweit er durch orthographische Konventionen beeinflusst wird. Zurecht fragt Schwegler (1990), ob frz. j'achète eher eine Einheit bilde als frz. je parle. Aus phonologischer Perspektive ist dies zu verneinen, aus graphematischer zu bejahen.1 D.h. eine solche Rechnung steht und fällt mit dem Wort- und mit dem Morphembegriff. So ergibt sich die weitere Frage, wie man mit Nullmorphemen umgeht. Zählt man bei nhd. Haus Nom. und Sg. mit - so wie man bei Häusern Dat. und Pl. dazurechnen würde? Wie verfährt man mit inhärentem Genus? Und wie vetrechnet man unterschiedliche Fusionsgrade innerhalb eines Wortes? Die ganz unterschiedliche Serialisierung der jeweils vier Informationen bei den Präterita starker und schwacher Verben, dargestellt in Abb. 2, würde bei der Greenbergschen Formel keinerlei Unterschied ergeben, d.h. in jedem Fall zu einem Syntheseindex von (mindestens) 4,0 führen (man könnte auch noch Modus dazuzählen).

Kaum eine Sprache wird auf der Analyse/Synthese-Skala so unterschiedlich verottet wie das Französische. Dies zu zeigen und zu erklären ist eins der Anliegen von Schwegle (1990), s. insbesondere die Zitatsammlung auf S.75-76

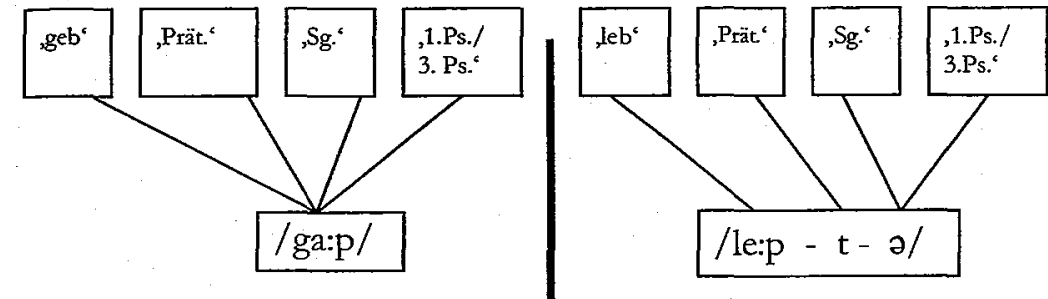

Abbildung 2: Vier Informationen in einem Wort bei unterschiedlicher Fusionienung

Es liegt auf der Hand, dass gerade für flektierende Sprachen wie das Deutsche solche Rechnungen unbefriedigend bleiben.

Nach Greenberg gab es weitere Versuche, Syntheseindexe zu erstellen, wobei sich die genannten Probleme als unüberwindbar erwiesen. Für diese Diskussion sei auf Schwegler (1990: 19ff.) verwiesen. So haben Lyons (1968) und Haarmann (1976) darauf hingewiesen, dass sich die Teilsysteme einer Sprache stark unterscheiden können: Pronominalsysteme sind oft sehr synthetisch, Nominal- und Verbalmorphologie können sich divergent verhalten.

\subsection{Die Kriterien nach Schwegler (1990)}

Schwegler (1990) schließt aus diesen Problemen, dass man den Syntheseindex von Sptächën glöbal nicht messen könne, sondern nur den von Teilsystemen. Auch seien Teilsysteme wegen des unscharfen Wortbegriffs sprachübergreifend kaum vergleichbar. Ein objektives, absolutes und sprachunabhängiges $\mathrm{Ma}$ sei nicht möglich. Am ehesten biete sich ein Vergleich zwischen unterschiedlichen historischen Stufen ein und derselben Sprache an oder zwischen miteinander verwandten Sprachen (z.B. den tomanischen Sprachen), die auf eine gemeinsame Sprache (Latein) zurückgehen. Was die Bezugsdomäne zur Ermittlung der Morphemzah betrifft, so entscheidet sich Schwegler angesichts der silbensprachlichen Verhältnisse des Französischen, sich auf sog. Sprecheinheiten (,speech units") zu beziehen. Hiermit dürfte das ,mot phonétique“ gemeint sein, oder ein definierter Komplex wie "Subjektspronomen + Verb", worunter sowohl die lateinischen als auch die französischen, italienischen etc. Konstruktionen fallen. Dies erlaubt es nach Schwegler (und entgegen Greenberg), auch grammatische Konstruktionen wie Periphrasen zu untersuchen. Auch sei es möglich, Kongruenz (als Fall morphologischer Interdependenz), die bisher nicht in solche Rechnungen einging, einzubeziehen. Letztendlich argumentiert Schwegler (1990) für die Berücksichtigung von vier Ebenen, die mehr oder weniger korrelativ miteinander verbunden sein können, es aber nicht müssen: 
Analyticity will be defined as the semantic, syntactic, morphological and phonological autonomy of morphemes within a speech unit. Syntheticity is characterized as the semantic, syntactic, morphological and phonological interdependengy (or relatedness) of morphemes witbin a speech unit. A unit whose morphemes show a high degree of relatedness on all four levels is highly synthetic; a unit whose morphemes have a very low degree of telatedness on all four levels is said to be highly analytic. (Schwegler 1990: 48; Kursivdruck im Original)

Dies lässt sich m.E. wie folgt schematisieren:

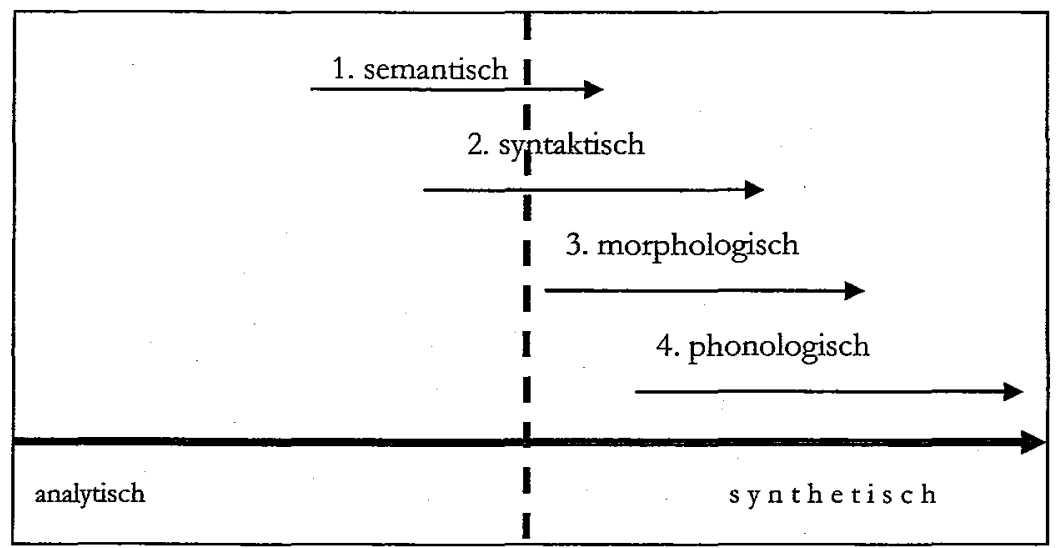

Abbildung 3: Die vier Ebenen dex Zunahme an Synthese nach Schwegler (1990)

Um von wirklich synthetischen Strukturen sprechen zu können (rechts des gestrichelten Senkrechtstrichs), müssen alle vier Parameter erfüllt sein; doch können durchaus schon semantische und syntaktische Synthesetendenzen wirken, ohne dass morpho-phonologisch synthetische Formen vorlägen. Insgesamt exinnern die Zusammenhänge an Grammatikalisierungsprozesse, die bei Schwegler jedoch noch kein Thema sind. Was die semantische Verdichtung steuert, sind die von Bybee (1985) beschriebenen Prinzipien der Relevanz und der Allgemeingültigkeit (,generality"), die hier nicht ausgeführt werden. Sie erweisen sich als zentral für die Frage, welche Informationen es grundsätzlich sind, die eine Nähe zueinander etlauben und damit langfristig auch einen synthetischen Ausdruck (s. auch Leiss 1998: 852).

Dass syntaktische, morphologische und phonologische Determinanten direkt die Linearität des Informationsausdrucks betreffen und verdichten, ist naheliegend. Bei der Bestimmung morphosyntaktischer Synthetizität verwendet Schwegler Kritetien wie Trennbarkeit, Fusionsgrad auf der syntagmatischen Achse, Verschiebbarkeit, Isolierbarkeit, Obligatorik sowie strukturelle und semantische Transparenz.
Wie die semantische Komponente witken kann, macht et anhand der Univerbierung der einst komplexen frz. Konjunktion parceque deutlich (s. Abb. 4): Bei Stufe 1 in Abb. 4 steht die Kombination dreier Funktionswörter zur Verfügung, par, ce und que, die jeweils ihre eigene Funktion entfalten, d.h. hier liegt ein analytisches, kompositionelles Stadium vor. In den Stufen 2-4 nimmt der Synthesegrad zu über die sukzessive Univerbierung durch (siehe oben Abb. 3) die syntaktische (z.B. Nichtunterbrechbarkeit der Folge), morphologische und phonologische Verdichtung (Auflösung der Wort- bzw. Morphemgrenzen), bis in Stufe 5 die totale Koaleszenz (Aufgabe sämtlicher Binnenstrukturen) erreicht wird - und damit wieder der Schritt zu einer analytischen, da intern nicht mehr analysierbaten Einheit: /parskə/. In diesem Fall handelt es sich um die Entstehung eines nicht flektierenden Grammems.

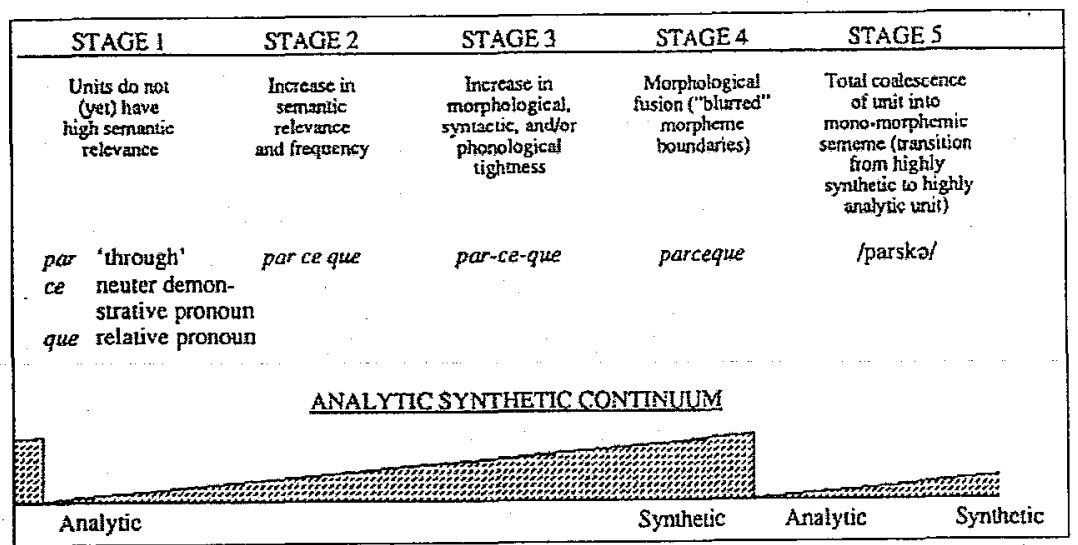

Abbildung 4: Der Zyklus von analytisch > synthetisch > analytisch anhand der Konjunktion parreque (aus Schwegler 1990: 50)

Semantische Koaleszenz kann jedoch auch bei syntaktisch noch selbständigen Einheiten gegeben sein. Hier ist an Funktionsverbgefüge, bestimmte Periphrasen oder an Partikelverben wie angeben, aufgeben zu denken, die syntaktisch in Distanzstellung treten können, aber semantisch eine feste lexikalische Einheit bilden (dies betrifft auch Pronominaladverbien - s. hierzu den Beitrag von Helmut Spiekermann in diesem Band).

Grundsätzlich gibt es nach Schwegler (1990) drei verschiedene Wege zur Analyse:

a) Abnahme an Synthetizität, z.B. indem Stammallomorphie ausgeglichen wird (hierzu s. später unter Abschnitt 3); 
b) totale Verschmelzung wie im Fall von parceque; auf das Deutsche bezogen z.B. nie /ni: / < ahd. ni eo, nicht jec oder nicbt / nixt/ < ahd. ni eo uuibt, nicht itgendeine Kleinigkeit;

c) Ersetzung einer synthetischen durch eine analytisch(er)e Form, z.B. des lat. Perfekts amavi durch frz. $j^{\prime} a i$ aimé oder nhd. käme durch würde kommen.

Schwegler (1990) kritisiert auch die oft angenommene Unidirektionalität bei Verschiebungen auf der Analyse/Synthese-Achse. Auch hier seien, oft innerhalb desselben Teilsystems, gegenläufige Entwicklungen festzustellen. Dies hänge damit zusammen, dass Verschiebungen auf dieser Achse nicht Ursache, sondern Folge vielfältigen Sprachwandels seien.

\subsection{Phänomene syntagmatischer Verdichtung:}

\section{Isolierende, agglutinierende und flektierende}

Ausdrucks- und Komprimierungsverfahren

Vor diesem Hintergrund erweist sich, dass sich die gängigen morphologischen Typen der isolierenden, agglutinierenden und flektierenden Sprachen hauptsächlich aus ihrem unterschiedlichen Synthesegrad ergeben. Dies visualisiert, angelehnt an Ronneberger-Sibold (1980), Abb. 5, wobe hier ein weiterer Typ, das sog. kombinierende (oder diskontinuierende) Verfahren, gleich hinzugefügt wird, doch erst unter 2.5 diskutiert wird.

Im Fall der isolierenden Strukturen entspricht einem freien Ausdruck, idealerweise einem Wort, genau eine Information. So drückt der engl. Artikel the nur Definitheit aus, nichts mehr. Eine solche 1:1-Entsprechung zwischen Ausdruck (,A") und Inhalt (Kategorie $=$ „, $\mathrm{K}^{\circ}$ ) sieht auch die Agglutination vor, doch verbinden sich dabei A1 und A2 zu einem Wort bei morphologischer Segmentierbarkeit. Dass bei agglutinierenden Sprachen ein möglichst geringer Grad an Allomorphie und Synkretismus hinzukommen sollte, bildet ein weiteres sprachtypologisches Kriterium, das hier ausgeklammert bleiben soll - ebenso, dass genau diese beiden Phänomene üblicherweise bei flektierenden Sprachen vorkommen. Für uns ist beim flektierenden Verfahren nur von Belang, dass sich der Informationsausdruck so stark überlagert, dass Portmanteau-Strukturen entstehen Hierfür liefert der deutsche Definitartikel ein Paradebeispiel: Bei $\{d\}-\{a s\}$ kodiert $\{d\}$ zwat allein (agglutinierend) Definitheit, doch lagern auf $\{a s\}$ die drei Informationen Genus, Numerus und Kasus. Als agglutinierend kann auch das Dentalsuffix - $t$ - im Deutschen gelten (wie bei leb-t-e in Abb. 2), wenn man der Analyse folgt, die Präteritalinformation nur auf dem $\{t\}$ kodiert sehen zu wollen, während der Suffixrest Person/Numerus markiert. Das kombinierende Verfahren kommt unter 2.5 zur Sprache.

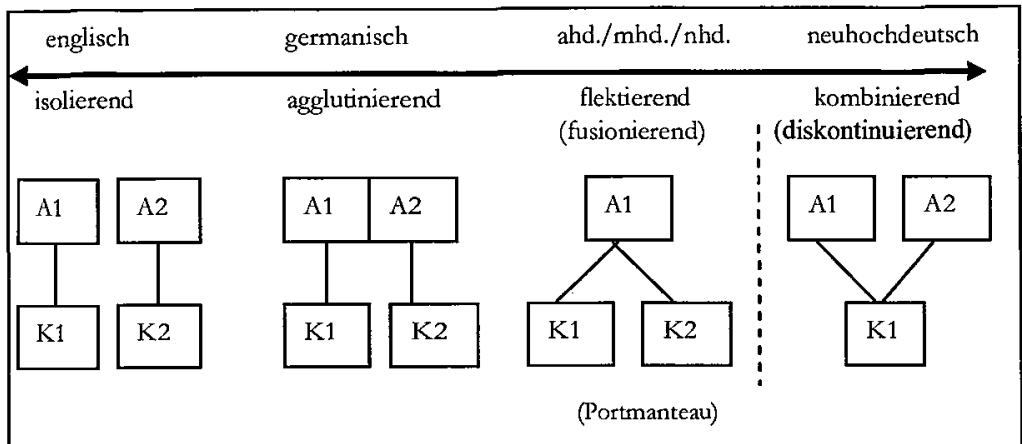

Abbildung 5: Die wichtigsten morphologischen Sprachrypen (Anmerkung: „A“ $\mathrm{A}^{\text {" }}$ Ausdruck, „K“ = Kategorie)

\subsection{Ein spezielles Phänomen syntagmatischer Verdichtung:}

Kongruenz

Schwegler überschreitet durch seine Bezugsgröße speech unit die Einzelwortgrenzen. Dabei stellt sich die interessante Frage, wie Kongruenz sich bezüglich der Frage nach dem Synthesegrad verhält. So steht der engl. Plural-NP the red bouse-s das spanische Pendant la-s casa-s roja-s gegenüber, wo jede Einheit, auch wenn teilweise redundant, am Pluralausdruck teilhat. Da bei Kongruenz die syntagmatische Binnenverdichtung, d.h. die morphologische Interaktion zwischen den einzelnen Bestandteilen zunimmt, wertet Schwegler dies als verstärkten Synthesegrad, der in die Rechnung eines Indexes eingehen sollte. D.h. je mehr Kongruenz, desto synthetischer. Ob es dabei einen Unterschied ausmacht, wenn, wie in dem spanischen Beispiel, die kongruierenden Affixe homophon sind oder nicht, soll unter Punkt 3 wieder aufgegriffen werden.

2.5 Ein speziell deutsches Phänomen syntagmatischer Verdichtung: Das kombinierende (diskontinuierende) Verfahren

Aus Kongruenz kann diachron Diskontinuität erwachsen: Genau dies ist das Anliegen von Werner (1979). Dabei entsteht dieses im Deutschen reich vertretene morphologische Kodierungsverfahten durch einstige synkrestisch, d.h. ambig gewordene Kongruenzaffixe: Die Affixe wiederholen hicht redundant gleiche Informationen, sondern sie eröffnen ambige Optionen, die erst durch weitere Affixe vereindeutigt werden (hierzu s. ausführlich Szczepaniak in diesem Band). Dies ist das letzte in Abb. 5 dargestellte Verfahren. Wie das Schema zeigt, verhält es sich invers zum flektierenden Verfahren: Die Verletzung der 1:1-Relation zwischen Ausdruck und Kategorie wird gespiegelt. Hier sind es zwei Ausdruckseinhei- 
ten, die erst im Verbund - „kombinierend“ (nach Ronneberger-Sibold 1980) - die Information K konstituieren. Die Bezeichnung „diskontinuierend" (Wemer 1979) bezeichnet das gleiche, betont jedoch im Gegensatz dazu die materielle Symbolisierung, d.h. dass sich die Morpheme in Distanzstellung befinden. Unter Umständen können die beiden ambigen Ausdrücke sogar beträchtliche Klammerkonstruktionen bilden, d.h. ausgedehnte Mittelfelder umfassen, etwa wenn zwischen Artikel und Substantiv längere Attribute tteten: der [kleinen und noch nicht stubenreinen] Hunde/ Katze/Katzen. Die hochgradig ambige Artikelform der eröffnet vier potentielle Lesarten:

1) Nom.M.Sg.

2) Gen.Fem.Sg

3) Dat.Fem.Sg.

4) Gen.Pl.

Ebenso sind auch die Substantive und die Adjektive hochgradig ambig. Option 1), Nom.M.Sg., wird durch die Flexion des Adjektivs verworfen. Die anderen drei Optionen werden erst durch das Kernsubstantiv vereindeutigt (zur Nominalklammer und ihrer Entstehung s. RonnebergerSibold 1991, 1994). Schematisch und vereinfacht zeigt dies Abb. 6, oben für die NP, unten für die VP.

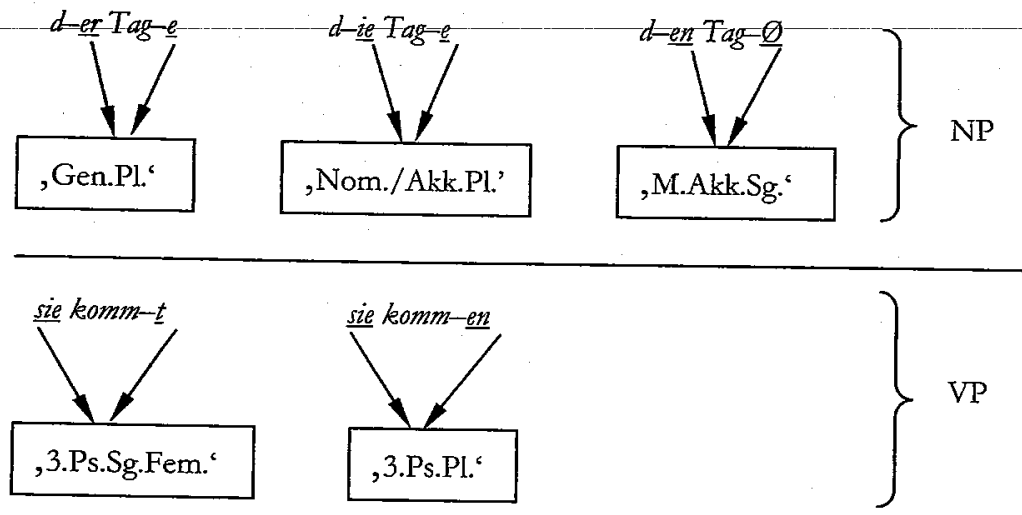

Abbildung 6: Diskontinuität als kombinatorisch erfolgender Informationsausdruck

Der entscheidende Unterschied der Diskontinuität zur Kongruenz liegt in der Ambiguität beider Elemente. Ein Fall von Kongruenz besteht dagegen bei du komm-st, er komm-t oder auch wir komm-en: Hier übernimmt bereits das Subjektspronomen die eindeutige Kategorienanzeige, die durch das
Verb nur nochmals aufgenommen wird (s. Abb. 7). Die Information 2.Ps.Sg. wird bereits durch $d u$ in eindeutiger Weise ausgedrückt.

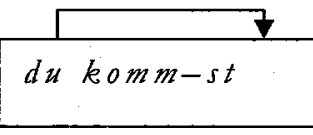

Abbildung 7: Kongruenz als Wiederaufnahme einer Information

Dass die kombinierend kodierten Informationen wiederum ganze Kategorienbündel beinhalten, zeigt, wie komplex das Deutsche organisiert ist. Maßgeblichen Anteil an dem Übergang vom kongruierenden zum diskontinuierenden Typ hatte die mhd. Nebensilbenabschwächung. Das Besondere ist jedoch, dass sich daraus kein rudimentäres Kongruenzverhalten herausgebildet bzw. die Kongruenz - wie im Englischen - komplett abgebaut wurde, sondern dass das kombinierende Verfahren weiter ausgebaut wurde und wird, auch auf periphrastischer Ebene (s. Abb. 8). Besonders hier wird deutlich, dass - bezogen auf Abb. 3 und die vier sprachlichen Ebenen - auf der semantisch-funktionalen Ebene bereits ein hoher Synthesegrad herrscht: Die Information ,Passiv' ergibt sich erst durch die Kombination aus einer Form von werden + dem Partizip II. Stünde hier ein Infinitiv, so ergäbe sich kombinatorisch die Futurlesart: sie wird seben. Vor diesem Hintergrund wird deutlich, wie unangemessen die häufig praktizierte Gleichsetzung von Periphrase und „analytischer Verbform “" ist (so z.B. in Polenz 2000, Bd. 1, S. 188).

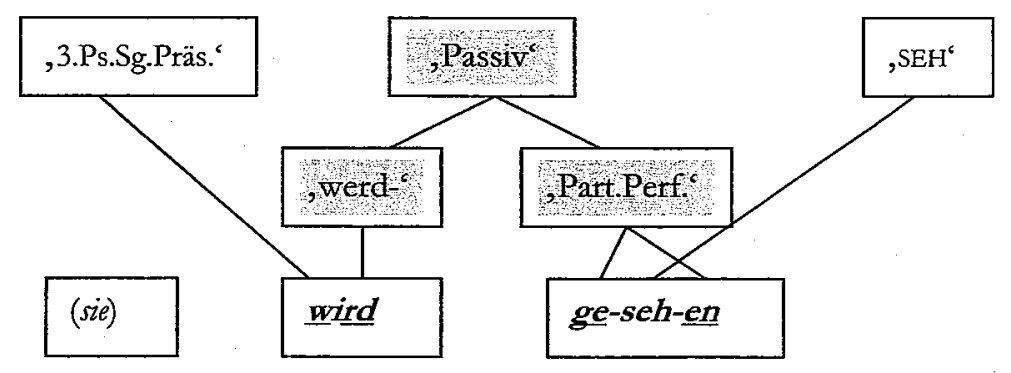

Abbildung 8: Diskontinuität als morphosyntaktisches Prinzip im Deutschen

Dass das kombinatorische Prinzip auch in Dialekten, die synkretistische Pronominalparadigmen enthalten, diachron gestätkt wird, weist Rabanus (2006) nach: In nordbairischen Dialekten kommt es in den Personalpronomen der 2.Ps.Pl. zu totalem Synkretismus, d.h. die Form enk (regional auch enks) deckt alle drei Kasus Nom., Dat. und Akk. ab (vgl. im Standard 
ibr Nom. vs. euch Dat./Akk.). In genau solchen Synkretismusgebieten „springt" das Verb „ein“, das in der 2.Ps.Pl. eine ts-Endung grammatikalisiert hat (die sich, entgegen dem Standard, von der 3.Ps.Sg. -t unterscheidet). Die ts-Endung klärt, dass es sich bei enk um den Nominativ, also das Subjektspronomen, handeln muss und nicht um einen Obliquus (Objekt) Der Satz enks (2.PI.Nom./Dat./Akk.) baes-tis (BEISSEN-2.PI.) dei (3.Sg./ Pl.Nom./Akk.) kann wegen der dis-ambiguierenden Verbendung nur als ,ihr beißt sie übersetzt werden, während enks baes-n (BEISSEN-1./3.PI.) det nur ,euch beißen sie / sie beißen euch' bedeuten kann. In jedem Fall ist es das Verb, das, im Verbund mit dem synkretistischen Pronomen, die Ambiguität ,auflöst" bzw. diskontinuierend markiert.2 Rabanus kann sogat dokumentieren, dass sich zwischen 1887 und 1999 das kombinierende Verfahren geographisch ausgebreitet, also verstärkt hat.

Die zentrale Frage, die sich abschließend stellt, ist, ob das diskontinuierende (kombinierende) Verfahren als synthetischer zu gelten hat als das flektierende. Mit Blick auf das unter 2.4 zur Kongruenz Gesagte ist dies eindeutig zu bejahen: Durch das kombinierende Verfahren nimmt die syntagmatische Binnenverdichtung, die morphologische Interdependenz noch ungleich stärker zu als bei der bloßen Kongtuenz als einem Wiederholungsphänomen; beim kombinierenden Verfahren werden nicht einfach nur bereits gesetzte Informationen repliziert, sondern überhaupt erst konstituiert. Damit hat, bezogen auf die sinnvollete Domäne der „speech unit" (als morphosyntaktischer Konstruktion), das kombinierende Verfahren als noch synthetischer zu gelten als das flektierende. Auf die bloße Wortebene bezogen würde das kombinierende Verfahren überhaupt nicht erfasst. Bei der mechanischen Anwendung der Greenbergschen Formel bliebe ein großer Teil der deutschen Morphologie unberücksichtigt.

\section{Analytizität bzw. Synthetizität als paradigmatisches Phänomen}

Nachdem einige wichtige Phänomene und Probleme auf der syntagmatischen Achse thematisiert worden sind, soll diskutiert werden, ob auch Phänomene auf der paradigmatischen Achse Einfluss auf die Synthetizität haben können. Schwegler (1990) stellt (nicht als erster) die Frage (und beantwortet sie positiv), ob auch Allomorphie eine Komponente bei der Ermittlung des Synthesegrads darstellt. Auf den ersten Blick erscheint dies nicht naheliegend, da Allomorphie als solche die Informationsabfolge und

$2 \quad$ Allerdings ist hier nur eine Einheit ambig (der funktionale Kopf, das Pronomen, während die Verbendung monofunktional ist), was das diskontinuierende Verfahren leicht einschränkt. -dichte nicht tangiert (es sei denn, Umlaut kommt ins Spiel). Auch die Greenbergsche Syntheseindexformel enthält sie nicht. Nach Schwegler steigert jegliche Form von Allomorphie - sei sie phonologisch, morphologisch, syntaktisch, semantisch oder lexikalisch konditioniert - den Synthesegrad, weil sie die strukturelle Interdependenz zwischen sprachlichen Einheiten verstärkt: Allomorphie ist meist von der strukturellen Umgebung, in der sie auftritt, abhängig. So ist die deutsche Pluralallomorphie direkt abhängig vom Genus des Substantivs (Teil des lexikalischen Eintrags), manchmal auch von seiner Silbenzahl, Betonung, seinem Auslaut, von der Belebtheit des Referenten etc. Stehen die Allomorphe in formal suppletivem Verhältnis zueinander, treibt dies den Synthesegrad nochmals in die Höhe. Auf die Pluralallomorphe bezogen: Die Allomorphe en und -n lassen sich unter das Pluralallomorph $\{(e) n\}$ subsumieren, da sie komplementär distribuiert und vom Auslaut abhängig sind: Schwache Feminina auf $-e$ erhalten $\{n\}$ (Blume-n), solche auf Nicht-Schwa $\{e n\}$ (Schrift-en, Idee-[a]n). Suppletiv verhält sich dagegen die Endung $\{(e) n\}$ der schwachen im Vergleich zum Verfahren $\{$ Umlaut $+-e\}$ der starken Feminina (Fluchten vs. Ausflücbte ). Dies steigert die Synthetizität.

A high level of syntheticity is reached when a morpheme has an abundance of allomorphs whose alternations can be captured only by a multitude of rules. Furthermore, allophones [sic - gemeint ist: allomophs] which bear no phonological resemblance to each other (cf. for instance Fr [frz.] je to mot) will be considered mote synthetic than those with extensive formal similarities (cf. $\mathrm{Fr} / \mathrm{vu} /$ to / vuz/). (Schwegler 1990: 64).

Indem Allomorphie oft auf bestimmte Kategorien(kombinationen) beschränkt ist, leistet sie auch einen Beitrag als sog. Nebenmarker. Dieser Terminus stammt von Wurzel (z.B. 1996) und steht dem sog. Hauptmarker gegenüber. So gilt das Verbalsuffix $-e[$ [a] gemeinhin als Person/Numerusmarker (1./3.Sg.): geb-e, leb-e, leb-t-e. Im Präteritum der starken Verben (und im Präsens der Präteritopräsentien) erscheint hier jedoch das sog. Null-Allomorph: gab- $\varnothing$, fiel- $\varnothing$ bzw. kann- $\varnothing$, muss- $\varnothing$. Da diese beiden Allomotphe flexionsklassen- und tempusspezifisch distribuiett sind (und genau dadurch eine dichtere, syntheseverstärkende Kohäsion an den Tag legen), tragen sie, wenngleich nur indirekt, auch zum Ausdruck eben dieser Informationen bei. Auch solche (im Deutschen übrigens zahlreiche) Nebenmarker müssten in eine Syntheseindexrechnung einbezogen werden. Wie sich das komplizierte Verhältnis zwischen Haupt- und Nebenmarkern in der deutschen Sprachgeschichte verschiebt, wird in Wurzel (1996) gezeigt.

Auch innerhalb kongruierender Suffixe kommt es oft zu Allomorphie: klein- Kälb-er renn-en berum. Dies hat als synthetischer zu gelten als Kongruenz mit homonymen Suffixen (wie im o.g. span. Bsp. la- $\underline{s}$ casa-s roja-s ). 
Schließlich gibt es auch in Periphrasen Allo-Phänomene, vgl. etwa die sein/baben-Distribution und -Bedingtheit in der deutschen Perfektperiphrase.

Wenn Allomorphie den Synthesegrad erhöht, muss konsequenterweise der Abbau von Allomorphie den Analysegtad erhöhen. Auch dem trägt Schwegler Rechnung; hier fühtt er den analogischen Stammalternanzausgleich von span. puedo - podemos in südamerikanischen Varietäten zugunsten des Diphthongs an (zu puedo-puedemos). Auf das Deutsche bezogen ist der Abbau von Wechselflexion als Analyseschub zu bewerten Indem z.B. backen derzeit die Formen bäck-st, bäck-t in back-st, back-t überführt, wird das gesamte Paradigma ärmer an Stammallomorphie und dami auch insgesamt analytischer. Damit ist das unter Abschnitt 2.2 erwähnte Verfahren a) (das einen Weg von drei möglichen zu vermehtter Analytizität beschreibt) gemeint und etklärt.

Synkretismus als intraparadigmatische Homonymie gilt konsequenterweise als analysesteigerndes Phänomen. Das heißt, starke Differenzierung steigert den Synthesegrad, mangelnde Differenzierung den Analysegrad. Allerdings ermöglicht Synkretismus auf der syntagmatischen Achse das kombinierende Verfahren und ist insofern an synthetischen Verfahren beteiligt.

Als Fazit ist festzuhalten, dass auch Allomorphie als paradigmatisches Phänomen den Synthesegrad positiv beeinflusst.

\section{Weitere Patameter zut Bestimmung von Analytizität bzw.}

\section{Synthetizität}

Auch jenseits der syntagmatischen und paradigmatischen Achse gibt es Parameter, die in die Waagschale geworfen werden müssen. Wie gezeigt, verfahren die starken Verben, da fusionierender, synthetischer als die eher agglutinierend organisierten schwachen Verben. Blickt man auf die Anzah starker und schwacher Verben im Deutschen, tut sich ein starkes Gefälle auf: Maximal 160 starken (und unregelmäßigen) stehen Tausende schwacher Verben gegenüber. Dies ergibt ein prozentuales Type-Verhältnis von 4,7 zu 95,3\% (nach Augst 1975, Nübling et al. 2008). Dies könnte dazu verleiten, dem Deutschen, wollte man den Synthesegrad der Verben bestimmen, nur die prototypischen schwachen Verben zugrundezulegen. Bekanntlich haben die Types jedoch nicht viel mit den Tokens gemein, of genug verhält sich ihr Vorkommen umgekehrt proportional zueinander. Dies gilt auch für die Verbklassen: Zählt man die Verben eines Fließtextes, überragen sogar die starken und irregulären Verben die schwachen; das Token-Verhältnis beträgt 59\% (nichtschwache Verben) zu $41 \%$ (schwache Verben). Dies zeigt, dass man bei einem Syntheseindex auch das faktissche Vorkommen, die Aktualisierung der gezählten Einheiten zu berücksichtigen hat und nicht nur die Types zählen kann.

Eine weitere Frage betrifft die Anzahl grammatischer Kategorien, die von Sprache zu Sprache differieren kann: So ist Aspekt im Deutschen keine Flexionskategorie, und innerhalb der Numeruskategorie gibt es keinen Dual. Andere Sprachen haben Definit-/Indefinitheit nicht grammatikalisiert. Je höher die Anzahl grammatischer Kategorien, desto höher, zumindest tendenziell, dürfte auch der Synthesegrad sein. Dies erschwert die Erstellung eines interlingualen Syntheseindexes.

Selbst wenn man den Syntheseindex nur auf der Basis von Wörtern ermitteln möchte - was im Deutschen als Wortsprache legitimer und ein facher ist als im Französischen als Silbensprache - so stellt sich die Frage, ob nut die eine Domäne der Morphologie, die Flexion, zugrundezulegen ist oder ob auch die Wortbildung einzubeziehen wäre, und, wenn ja, wie und mit welchem Gewicht. Wurzel (1993) unterstellt der deutschen Wortbildung sogar inkorporierende Strukturen, d.h. insgesamt dürfte die Wortbildung angesichts dessen, aber auch angesichts der Kompositionsfreudigkeit und der Herausbildung neuer Affixe aus Affixoiden den Syntheseindex nach oben treiben. Darauf nimmt auch Roelcke (2002: 340) Bezug, wenn er schreibt:

So findet nämlich der Abbau der synthetischen Bauweise vornehmlich im Beteich der Formbildung statt, während sich der Ausbau der synthetischen Bauweise insbesondere auf den Bereich der Wortbildung erstreckt. [...].

\section{Einige Fallbeispiele aus der deutschen Sprachgeschichte}

Der für das Deutsche typologisch kennzeichnende Kategorienausdruck des kombinierenden Verfahrens kam bereits ausführlich zur Sprache. Abschließend sollen schlaglichtartig vier weitere einschlägige Beispiele aus der deutschen Sprachgeschichte beleuchtet werden, die für die Drift zu mehr Synthese sprechen.

\subsection{Der morphologische Umlaut}

Das Deutsche hat für den Ausdruck von mindestens drei grammatischen Kategorien das Umlautverfahren morphologisiert: Den nominalen Plural (Habn - Häbne), den adjektivischen Komparativ (gesund - gesïnder - gesünde sten) und den verbalen Konjunktiv II (singt - sänge). Dass der Umlaut eine lautgesetzliche Basis hat, ist bekannt, ebenso dass er durch seinen modifikatorischen Charakter einen extrem fusionierenden Marker ohne materiellen Mehraufwand darstellt. Nur im Deutschen (und Luxemburgischen) hat 
seine Morphologisierung (Grammatikalisierung) stattgefunden. Beim Substantiv hat er sich aus den angestammten Klassen gelöst (hier: die maskuline und feminine $i$-sowie die neutrale $i ₹ / a \nsucc$-Klasse) und ist analogisch auf Mitglieder anderer (umlautloser) Klassen übertragen worden. Ähnliches gilt für die Adjektivsteigerung, und auch bei den Verben erweist sich seine Morphologisierung anhand der Tatsache, dass er beim präteritalen Numerusausgleich im Frühnhd., also viele Jahrhunderte nach seiner lautgesetzlichen Phàse, aüf die neuen Einheitspräterita projiziert wurde: frühes frühnhd. singen - sang (Prät.Sg.) - sungen (Prät.Pl. + Basis für Konj.II) gesungen $>$ spätes frühnhd. singen - sang(en) - gesungen. Die alte Prät.Pl.-Form sungen, die auch die Basis für die gesamte Konj. II-Bildung stellte (ich/sie sünge, wir/sie süngen etc.), wurde aufgegeben. Der bis dahin längst morphologisierte Konjunktivumlaut wurde daraufhin auf den neuen Präteritalstamm sang- übertragen: ich/sie sänge, wir/sie sängen etc. Alle eingangs genannten Beispiele betreffen solche analogischen Umlaute. Besondere Erwähnung verdient der reine nominale Pluralumlaut wie z.B. bei Laden Läden, Boden - Böden, der sich erst später herausgebildet hat und noch bis heute leicht produktiv ist (vgl. derzeitige Schwankungsfälle wie die Pfosten/ Pfösten, die Wragen/Wägen, die Sattel/Sättel, die Ranzen/Ränzen).

Durch diese (morphologisch motivierten) Reanalyseprozesse ist das Deutsche zu einem hohen Synthesegrad gelangt. Da, wie Köpcke (1993) nachweist, es gerade tokenfrequente Substantive sind, die den Pluralumlaut übernommen haben, erhöht dies seinerseits die Synthetizität.

Dieses besonders dichte Syntheseverfahren ist auch deshalb interessant, weil Schwegler "nur" phonologisch sich manifestierende Synthesearten unberücksichtigt lässt. In den romanischen Sprachen kommen solche immateriellen Reanalysen anscheinend nicht vor. Phonologische Verdichtungen gehen nach Schwegler (1990) ,,automatisch“ vonstatten, da sie immer auf Lautgesetzen wie Assimilationen, Vokalharmonien, Reduktionen etc. beruhen. Das Deutsche liefert ein eindtucksvolles Beispiel dafür, dass sich Assimilationen, worauf der Umlaut ja zurückgeht, von jeglicher Lautgesetzlichkeit lösen und als grammatische Kategorie reanalysiert werden können, um so in die morphologische Domäne „aufzusteigen“, ohne segmenthaltige Affixe entwickeln zu müssen.

\subsection{Die Fragmentierung des Ablautverfahrens}

\section{als Zuwachs an Allomorphie}

Wir bleiben auf der morphophonologischen Ebene und rücken ein Phänomen in den Vordergrund, das normalerweise in die Irregularitätsdomäne verschoben wird und damit aus dem Blick gerät: Die einst fünf idg. Ablautreihen werden im Germanischen um eine sechste Reihe erweitert und im Ahd. um eine siebte. In der Folge erfährt dieses Ablautsystem eine starke Fragmentierung: Dies beginnt schon mit der Aufspaltung mancher Reihen im Ahd., was phonologischen Kontakterscheinungen (Hebung, Senkung, ahd. Monophthongierung mit kontextbedingten Ausnahmen etc.) geschuldet ist, die sich auf den Vokalismus auswirken. Diese Fragmentierung setzt sich über die Jahrhunderte fort und wird durch den präteritalen Numerusausgleich nicht eingedämmt, im Gegenteil. Nicht selten isolieren sich Verben durch singuläre Kontaktphänomene von ihrer ursprünglichen-Reihe-(z.B. nhd. kommen $<$ - ahd. queman). Oies-führt zu heute ca. 40 unterschiedlichen Alternanzen, darunter 15, die nur von einem einzigen Verb besetzt sind, z.B. k[j]mmen - k[a.]m - gek[j]mmen. Der größte Verband umfasst noch 23 Verben mit den Stammvokalen nhd. [ai - II], Typ reiten - ritt-geritten.

Diese aus Sicht des Gesamtsystems eingetretene Fragmentierung ist auf der anderen Seite nichts anderes als eine massive Zunahme an Allomorphie. Die Makroklasse der starken Verben, die Vokalwechsel für den Ausdruck von Tempus verwendet, hat sich in zahlreiche Mikroklassen bis hin zu Einzelgängern aufgespalten. Wie beim Umlaut handelt es sich auch hier um ein morphonologisches, extrem synthetisches Ausdrucksverfahren, das durch die Zunahme an Differenzierung auf der paradigmatischen Achse (Allomorphie) an Synthetizität gewinnt. Im Unterschied zum Umlaut, der eine klare, vorhersagbare Basisvokal $\rightarrow$ Umlaut-Relation beinhaltet, handelt es sich beim Ablaut um ein arbiträres, idiosynkratisches Vokalwechselverfahren, was seinerseits den Syntheseindex eher steigert als senkt.

\subsection{Analytischer Kasusausdruck?}

\section{Der Artikel als hochsynthetische Einheit}

Insbesondere der Kasusabbau am Substantiv und seine Verlagerung auf den Artikel gilt in den sprachgeschichtlichen Darstellungen als Paradebeispiel für einen Analyseschub (zu diesem Komplex s. Szczepaniak in diesem Band). Vergleicht man jedoch die Informationskodierungen zwischen dem Ahd. und Nhd. (s. Abb. 9), so macht das heutige Verfahren keinen analytischeren Eindruck. Richtig ist aber, dass Kasus nicht mehr am Nomen selbst markiert wird. Dies hängt, wie Primus (1997) herausarbeitet, mit der in der Geschichte des Deutschen vielfach zu beobachtenden Separierung von semantischem und funktionalem Kopf zusammen. Dabei wurden die funktionalen Köpfe linksperipher platziert und dort Đlexivisch oft sogar gestärkt, während die semantischen Köpfe am rechten Rand der Wortgruppe verblieben. Mit einem Analyseschub habe dies, so stellt Primus zutreffend fest, nichts zu tun. 


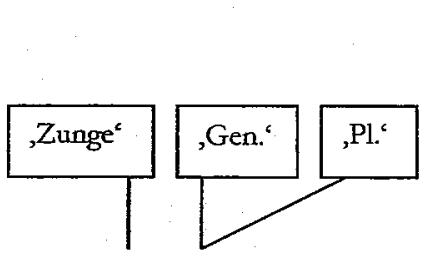

ahd. zung - ono

bbildung 9: Der Kasusausdruck früher und heu

Die Verhältnisse sind im Ahd. viel einfacher: Das stammflektierende Nomen zung-a realisiert die Kombination Gen.Pl. in einem Portmanteaumorphem. Nicht anders ist dies heute beim Artikelteil -er, der allerdings stark synkretistisch ist und die Information Gen.Pl. erst im Verbund (kombinierend) mit der Substantivendung $-n$ konstituiert (der allein evoziert vier verschiedene Lesarten). Die Grammatikalisietung des Definitartikels bereichert die NP um eine weitere Kategorie, , +definit'. Der Artikel dürfte wohl die synthetischste Einheit im Deutschen darstellen. Die Pluralinformation liegt weiterhin auf der Endung des Nomens und vereindeutigt die ambige Artikelform.

\subsection{Zum, zut, beim, ins, ans:}

Zur Grammatikalisierung „flektierender Präpositionen“

Bei der Analyse/Synthese-Diskussion wird mit aller Regelmäßigkeit einer der größten Syntheseschübe des Deutschen übersehen: Die sich schon im Ahd. anbahnende Verschmelzung von Präposition und Artikel. Es dürfte wenig übertrieben sein zu behaupten, dass es kaum einen Satz ohne eine solche Enklise gibt, d.h. dieses Phänomen kommt extrem häufig vor. Dabei erstrecken sich diese Amalgamierungen von allegrosprachlichen Verschmelzungen wie $a u f^{\prime} m$, nach' $m$, in' $n$ über einfache Klitika wie vorm, binters, übers bis hin zu obligatorisch klitisierten und nicht mehr (frei) auflösbaren Formen wie zum, beim, am, im, vom (vom $\neq$ von dem Bäcker, zum $\neq q^{u}$ dem Art, beim/*bei dem Singen, im/*in dem Schwarzwald, beim/*bei dem Papst, zu diesem Komplex s. Nübling 1992, 1998, 2005).

In Hinblick auf die Analyse/Synthese-Debatte ist wichtig, dass der overte Definitheitsmarker $d$ - durch den Klitisierungsprozess schwindet und ,Definitheit' damit auf das Artikelenklitikon tückt, denn in aller Regel bleiben diese Verschmelzungsformen zu ihren Pendants mit dem Indefinitartikel distinkt: vorm vs. vor'nem, binters vs. binter'n, beim vs. bei'nem, im vs. in'nem etc. Schematisch zeigt Abb. 10 diesen Komprimierungseffekt (dabei wurden Genus, Numerus und Kasus bereits zu einer Portmanteau-Einheit komprimiert).
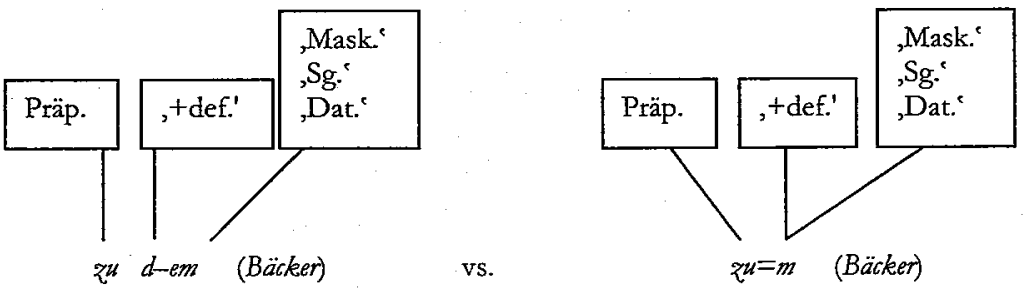

Abbildung 10: Der Syntheseeffekt der Präposition-Artikel-Enklise

Was die Tokenfrequenzrelationen zwischen Verschmelzung und Nichtverschmelzung betrifft, so wurde in Nübling (2005:113ff.) korpusbasiert gezeigt, dass die sechs Verschmelzungsformen am, zum, zur, im, vom, beim zu (teilweise sogar weit) über $90 \%$ vor den Nichtverschmelzungen tangieren (die im Fall von an dem, zu dem, zu der und in dem nur um die 2-3\% betragen). Gerade bei diesen sechs Verschmelzungsformen handelt es sich um die grammatikalisierteste "Spitze des Eisbergs“. Inwieweit es sich schon um flektierende Präpositionen handelt, wird in der genannten Literatur diskutiert. In jedem Fall steuert diese Grammatikalisierung auf dieses Szenario hin, was übrigens die allgemeine Beobachtung von Primus (1997) untermauert, dass linksperiphere funktionale Köpfe nicht nur zu Flexionserhalt bzw. zu Flexionsverstärkung tendieren: In diesem Fall handelt es sich sogar um Flexionsaufbau.

\section{Fazit}

Bei genauerem Hinsehen, was Analytizität und Synthetizität genau konstituiert, wird deutlich: Allzu oft handelt es sich um intuitive Bewertungen sprachlicher Strukturen, die selten objektiviert werden. Folgt man der romanistischen Untersuchung von Schwegler (1990), so wird hier ein sehr differenzierter, umfassender, die gesamte Forschung aufarbeitender und m.E. adäquaterer Synthesebegriff vertreten, der nicht nur die syntagmatische Achse der linearen Verdichtung berücksichtigt, sondern auch die paradigmatische Achse einbezieht. Dieses Geflecht fasst Abb. 11 zusammen. Auf beide Dimensionen bezogen dürfte das Deutsche diachron kaum an Synthetizität verloren haben, eher im Gegenteil. Dies wird in den meisten sprachgeschichtlichen Arbeiten anders bzw. vereinfacht dargestellt, da sie meist nur eindimensional ausgerichtet sind und oft von Unidirektionalität ausgehen. Hier wurde gezeigt, dass die diachronen Entwicklungen im Deutschen oft gleichzeitig in beide Richtungen laufen. 


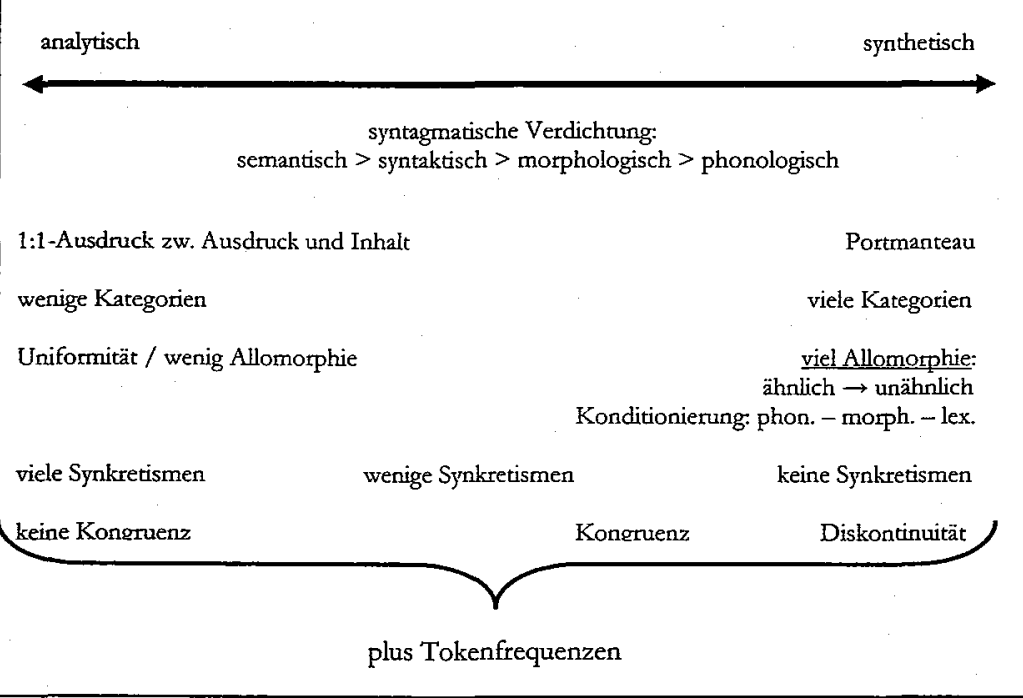

Abbildung 11: Abhängigkeit des Synthesegrads von (mindestens) folgenden Faktoren

Ein absoluter, einzelsprachunabhängiger Syntheseindex ist daher kaum ermittelbar. Man müsste dafür feste Maße für die Einzelphänomene, die hierfür eine Rolle spielen, bestimmen. Wie soll man z.B. syntagmatische Dichte mit Allomorphie verrechnen? Oder Portmanteau-Strukturen mit Kongruenz? Diskontinuität mit syntaktischer und/oder semantischer Fusion? Und wie sollte man mit alledem die Frequenzen verrechnen? „Wiegt" ein seltenes periphrastisches (kombinierendes) Futur etwa die viel häufigeren synthetischen Präterita auf?

Die im Beitrag genannten Faktoren sollten jedoch nicht dazu führen, sich von dem sog. Pendel zwischen Analyse und Synthese abzuwenden Ziel war es, zu einer differenzierteren Sicht auf die Komplexität dieses Unterfangens zu gelangen. M.E. sollte auch für das Deutsche die von Schwegler (1990) angemahnte Beschränkung auf einen nur relativen Vergleich geltend gemacht werden, der auch nur bezüglich eines einzelnen Parameters möglich ist und der nur feste sprachliche Einheiten (,speech units") betrifft. Dabei lassen sich am ehesten synchton zwei miteinander verwandte Sprachen oder diachron zwei verschiedene Sprachstufen kontrastieren.

\section{Literatur}

Augst, Gerhard (1975), Wie stark sind die starken Verben? Überlegungen zur Subklassifikation der nhd. Verben. In: Augst, Gerhard. (Hrsg.), Untersucbungen zum Morpheminventar der deutschen Gegenwartssprache. Tübingen: Nart, 231-281.

Bittner, Andreas \& Klaus-Michael Köpcke (in diesem Band), Ich mürde, wenn ich wüsste, dass ich könnte .... Der deutsche Konjunktiv zwischen Synthese und Analyse.

Bybee, Joan (1985), Morphology. A study of the relation between meaning and form. Amsterdam \& Philadelphia: Benjamins.

Greenberg, Joseph H. [1954] (1960), A quantitative approach to the morphological typology of language. International Journal of American Linguistics 26: 178-194.

Haarmann, H. (1976), Aspekte der Arealtypologie: Die Problematik der eurpäischen Sprachbünde. Tübingen: Narr. (Tübinger Beiträge zur Linguistik 72)

Köpcke, Klaus-Michael (1993), Schemata bei der Pluralbildung im Deutschen. Versuch einer kognitiven Morphologie. Tübingen: Narr.

Leiss, Elisabeth (1998): Ansätze zu einer Theorie des Sprachwandels auf morphologischer und syntaktischer Ebene. In: Besch, Werner Oskar Reichmann \& Stefan Sonderegger (Hrsg.), Sprachgeschichte, Bd. 1. Berlin \& New York: de Gruyter, 850860.

Nübling, Damaris (1992), Klitika im Deutschen - Schriftsprache, Umgangsppracbe, alemannische Dialekte. Tübingen: Narr.

Nübling, Damaris (1998), Wann werden die deutschen Präpositionen flektieren? Grammatisierungswege zur Flexion. In: Fabri, Ray, Albert Ortmann \& Teresa Parodi (eds.), Models of Inflection. Tübingen: Niemeyer, 266-289.

Nübling, Damaris (2005), Von in die über in'n und ins bis imr. Die Klitisierung von Präposition und Artikel als "Grammatikalisierungsbaustelle". In: Leuschner, Torsten, Tanja Mortelmans \& Sarah de Groodt (Hrsg.), Grammatikalisierung im Deutschen. Berlin \& New York: de Gruyter, 105-131.

Nübling, Damaris, Antje Dammel Janet Duke \& Renata Szeczepaniak (2008), Historische Spracbuissenscbaft des Deutschen. Eine Einfübrung in die Prinzipien des Sprachwandels. Tübingen: Narr.

Polenz, Peter von (2000), Deutsche Sprachgeschichte. 3 Bände. Berlin \& New York: de Gruyter.

Primus, Beatrice (1997), Der Wortoruppenaufbau in der Geschichte des Deutschen: Zur Präzisierung von synthetisch und analytisch. Sprachwissenschaft 22: 133-159.

Rabanus, Stefan (2006), An der Schnittstelle von Morphologie und Syntax. Einheitsformen der Personalpronomen der 1. und 2. Person Plural im Nordbairischen. Zeitscbrift für Dialektologie und Linguistik 73: 301-317.

Roelcke, Thorsten (1997), Sprachtypologie des Deutscben. Berlin \& New York: de Gruyter.

Roelcke, Thorsten (1998), Typologische Unterschiede in den Varietäten des Deutschen. In: Besch, Wemer, Oskar Reichmann \& Stefan Sonderegger (Hrsg.), Sprachgeschichte Bd. 1, Berlin \& New York: de Gruyter, 1000-1013.

Roelcke, Thorsten (2002a), Syntheseindex. Typologische Betrachtungen zum Deutschen in Synchronie und Diachronie. In: Wiesinger, Peter (Hrsg.), Zeitenwende die Germanistik auf dem Weg vom 20. ins 21. Jabrbundert. Bern: Lang, 337-342.

Roelcke, Thorsten (2002b), Sprachtypologische Tendenzen der deutschen Gegenwartssprache. Leuvense Bijdragen 91: 259-285. 
Ronneberger-Sibold, Elke (1976), Performanz und Sprachwandel. Zum Wandel vom ,flektierenden' zum isolierenden' Sptachtyp. In: Weber, Heinrich \& Harald Weydt (Hrsg.), Sprachtheorie und Pragmatik, Bd. 1. Tübingen: Niemeyer, 87-97.

Ronneberget-Sibold, Elke (1980), Sprachvenvendung - Spracbsystem. Ökonomie und Wandel. Tübingen: Niemeyer.

Ronneberger-Sibold, Elke (1991), Funktionale Betrachtungen zu Diskontinuität und Klammerbildung im Deutschen. In: Boretzky, Norbert, Werner Enninuitat und dikt Jeßing \& Thomas Stolz (Hrsg.), Spracbwandel und seine Prinzipien. Bochum: Brockmeyer, 206-236.

Ronneberger-Sibold, Elke (1994), Konservative Nominalflexion und „klammerndes Verfahren“ im Deutschen. In: Köpcke, Klaus-Michael (Hrsg.), Untersucbungen zur deutschen Nominal- und Verbalmorphologie. Tübingen: Narr, 115-130.

Schmidt, Karl Horst (1998), Versuch einer geschichtlichen Sprachtypologie des Deutschen. In: Besch, Werner, Oskar Reichmann \& Stefan Sonderegger (Hrsg.), Sprachgeschichte, Bd. 1. Berlin \& New York: de Gruyter, 993-1000.

Schmidt, Wilhelm (102007), Gescbicbte der deutschen Spracbe. Stuttgart: Firzel

Spiekermann, Helmut (in diesem Band), Pronominaladverbien im Niederdeutschen und in der norddeutschen Regionalsprache.

Schwegler, Armin (1990), Analyticity and syntheticity. A diachronic perspective with special reference to Romance languages. Berlin \& New York: de Gruyter.

Sonderegger, Stefan (1979), Grundzüge deutscher Sprachgeschichte. Diachronie des Sprachsystems. Berlin \& New York: de Gruyter.

Stedje, Astrid ( ${ }^{41999)}$, Deutsche Sprache gestern und heute. Einführung in Sprachgeschichte und Sprachkunde. München: Fink.

Szczepaniak, Renata (in diesem Band), Wird die deutsche Nominalphrase wirklich analytischer? Zur Herausbildung von Diskontinuität als synthetische Verdichtung.

Werner, Otmar (1979), Kongruenz wird zu Diskontinuität im Deutschen. In: Brogyanyi, Beia (eds.), Studies in Diacbronic, Syncbronic, and Typological Linguistics. Festschrift für Oswald Szemerényi, Bd. 2. Amsterdam \& Philadelphia: Benjamins, 959-988.

Werner, Otmar (1987), The aim of morphological change is a good mixture - not a uniform language type. In: Giacalone Ramat, Anna, Onofrio Carruba \& Giuliano Bernini (eds.), Papers from the 7th International Conference on Historical Linguistics. Amsterdam \& Philadelphia: Benjamins, 591-616

Werner, Otmar (1989), Sprachökonomie und Natürlichkeit im Bereich der Morphologie. Zeitschrift für Phonetik, Sprachwissenschaft und Kommunikationsforschung 42, 34-47.

Wurzel, Wolfgang Ullich (1996), Morphologischer Strukturwandel: Typologische Entwicklungen im Deutschen. In: Lang, Ewald \& Gisela Zifonun (Hrsg.), Deutsch - typologisch. IDS-Jabrbuch 1995. Berlin \& New York: de Gruyter, 492-524.

Wurzel, Wolfgang Ullrich (1993), Inkorporierung und ,Wortigkeit im Deutschen. In: Tonelli, Livia \& Wolfgang U. Dressler (eds.), Natural Morphology. Padova: Unipress, 109-125.
Andreas Bittner \& Klaus-Michael Köpcke

\section{Ich würde, wenn ich wïsste, dass ich könnte ... - Der Konjunktiv zwischen Synthese und Analyse}

\section{Abstract}

The aim of the present study was to verify two hypotheses on the use of synthetic inflexion in the German subjunctive (Konjunktiv II). Hypothesis 1 suggests that speakers adhere to the synthetic form. Hypothesis 2 considers forms which had lost their original function due to processes of language change. We propose their refunctionalisation as a method of identifying a form's more complex grammatical category. Data was gathered from speakers from both Northern Germany and Switzerland, where preterite-forms are disappearing. Results showed that the hypothesis of a general change from synthetic to analytic subjunctive forms cannot be supported Participants often made use of the potential for composing syntheic forms, whereby simple weak forms were allowed to compete with forms using specific ablaut or umlaut patterns, as well as "mixed" forms (singte/gerbte - sänge/gärbe - sängte/gärbte). Processes of grammaticalisation and reanalysis appear to result in a functionalisation of the last remaining morphological coding capacity for the identification of the most complex category. Preterite forms are reanalyzed as a synthetic designation of the subjunctive. Data suggest that synthetic inflexion in German subjunctive morphology is based on pattern or schema recognition and use.

\section{Ausgangspunkte und Gliederung}

Der folgende Beitrag wird sich mit dem deutschen Konjunktiv II beschäftigen, der Konjunktiv I findet nur am Rande Beachtung. Nach einem knappen Verweis auf das Formeninventar werden wir zwei Hypothesen entwickeln, die wir anhand empirischer Daten aus Tests mit Sprechern aus dem norddeutschen und aus dem schweizerdeutschen Sprachraum verifizieten wollen. Gesucht werden Antworten auf Fragen nach den Chancen und Bedingungen für eine synthetische Bildung des Konjunktiv II und seinem Verhältnis zu den sogenannten analytischen ,Ersatzformen'. Dieses Ziel impliziert auch Aussagen über das Vothandensein eines Tempusbezugs und die Ableitungsbasis der Konjunktivformen. Abschließend wollen wir eine theoretische Einordnung skizzieren und spekulieren, wie der Konjunktiv in möglicherweise nicht allzu ferner Zukunft gebildet werden könnte.

Die synthetischen Konjunktivformen sind ein prototypischer Übergangskandidat von der synthetischen zur analytischen Konstruktion. Die analytischen Bildungen des Konjunktivs sind tradiert und im Sprachge- 


\section{Linguistik - Impulse \& Tendenzen}

Herausgegeben von

Susanne Günthner

Klaus-Peter Konerding

Wolf-Andreas Liebert

Thorsten Roelcke

34

\section{Kodierungstechniken im Wandel}

Das Zusammenspiel von Analytik und Synthese im Gegenwartsdeutschen

Herausgegeben von

Dagmar Bittner und Livio Gaeta 


\section{Vorwort}

Dieser Sammelband geht auf eineg Workshop zurück, det im Februar 2008 unter dem Titel Das ewrige Pendel von synthetisch zu analytisch qu synthetisch ... - aktuelle Sprachwandeltendenzen im Rahmen der 3. Tagung „Deutsche Sprachwissenschaft in Italien" stattfand. Ziel des Workshops wat es, aktuelle Verändenungsprozesse im Deutschen unter dem Aspekt der sich wandelnden Kodierungstechniken zu diskutieren. Das Interesse, das der Workshop er hat, führte zu der Überlegung, dieses Thema im Rahmen eines Sammelbandes weiter zu erörtern.

Unsere Ausgangsfeststellung war, dass gegenwärtig verschiedene, z.T. schon lange angelegte Veränderungsprozesse gleichzeitig die Wahrnehmungsschwelle der normativen Grammatikschreibung übertreten. Man kann u.E. aber nicht nur in Hinsicht auf die Wahrnehmung sondern auch in Hinsicht auf die Akzeptanz und den Grad der Durchsetzung verschiedener Veränderungen von einem Eintritt in eine kulminierende Phase des Sprachwandels sprechen. Dabei stellt sich natürlich die Frage, was sich genau geändert hat bzw. ändert - und was eben nicht, wie Vilmos Ágel in seinem Beitrag betont. Zunächst und offensichtlich sind es die Kodierungstechniken, die Änderungen unterliegen. Weniger offensichtlich, aber durch die Grammatikforschung zu klären ist, inwiefern dies auf eine Änderung von generellen Strukturierungsprinzipien verweist und ob und welche Veränderungen in den Kategoriensystemen daraus resultieren.

Die adäquate Erfassung der Veränderungsprozesse ist u.E. aus mindestens drei Perspektiven zu leisten.

Aus empirischer Perspektive sind die einzelnen Veränderungsprozesse, aber vor allem die Momente ihres Zusammenwirkens, ihre eventuelle gegenseitige Abhängigkeit detailliert zu erfassen. $\mathrm{Zu}$ diesen Prozessen gehören morpho-syntaktische Veränderungen in der Realisierung von Aspekt, Tempus und Modus (vgl. u.a. Funktionsverbgefüge, Progressiv, Ersetzung des Präteritums durch das Perfekt, Ersetzung bzw. Wegfall des Konjunktivs), Abbautendenzen beim Artikel und daraus resultierende Prozesse (vgl. u.a. die Zunahme inkorporierender Prädikate, syntaktische Kasusrealisierung, flektierte Präpositionen) sowie auch tein syntaktische Veränderungen wie die Verbzweitstellung in weil-Sätzen und die Distanzstellung von Pronominaladverbien.

Aus grammatiktheoretischer Perspektive werfen diese Verändenungen Fragen hinsichtlich der Reorganisation des grammatischen Funktionsgefüges und der Veränderung von Kodierungstechniken auf. Letzteres ist im Deutschen ein besonders brisanter Aspekt, weil die sogenannte Klammerstruktur - die mit Distanzstellung verbundene analytische Kodierungstechnik - in der germanistischen Linguisten als besonderes typologisches

(C) 2010 Walter de Gruyter GmbH \& Co. KG, Berlin/New York

Druck: Hubert \& Co. GmbH \& Co. KG, Göttingen

Printed in Germany

www.degruyter.com

\section{e-ISBN 978-3-11-022845-8}

Bibliografische Information der Deutschen Nationalbibliothek

Die Deutsche Nationalbibliothek verzeichnet diese Publikation in der Deutschen Nationalbibliografie; detaillierte bibliografische Daten sind im Internet 